\title{
The Use of Simulation in Training to Improve Motor Learning Skill Acquisition for Complex Tasks in the Medical Industry
}

\section{Hannah $\mathbf{M}^{*}$ \\ School of Human Movements, University of Queensland, Australia}

*Corresponding author: Hannah L Morgan, Aspec Engineering Pty Ltd, University of Queensland School of Human Movements, Australia, Email: info@hannahhumanfactors.com

\section{Research Article \\ Volume 3 Issue 3}

Received Date: April 15, 2019

Published Date: May 07, 2019

DOI: $10.23880 /$ eoij-16000205

\section{Abstract}

For years, training across various industries has involved on the job or classroom-based training. In the medical industry they primarily adopt the Halstedian method of whole-task motor learning training. This has been found in the literature to lead to slower learning training times resulting in higher learning costs, resources and more opportunity for human error. It assumes the premise that instruction improves performance, which has also been found to be less effective to reduce human error in highly complex settings compared with other training modes. This is one of several studies which examines the benefits of the use of simulation to enhance trialing various modes of training, enhance skill acquisition and user acceptance of participants. This experiment quantifiably explores how the use of a non-fidelity simulator for colonoscopy training improved the learning curve of participants. Lower cost simulators have been found to reduce costs but allow for training modification to enhance the quality of training. In addition, often the low fidelity models have been found to have equal learning transference as higher fidelity models. Of all surgeries, colonoscopies has a four year learning curve. It is an ergonomically a difficult technical skill to master ie simultaneously performing the diagnostic examination and surgery removal of matter. By conducting a task analysis, separating the task of use of equipment and diagnostic tasks, it was found that they require vastly different skills. The study examined if skill acquisition can be achieved using a low fidelity simulator to enhance the learning curve of the whole set of tasks. Using university medical students across various cases using the simulator demonstrated that this was the case. In addition, self-reporting of students found that the use of the simulator enhance confidence in performing the task on a patient. Further research should explore this further to examine the impact on surgical performance and human error.

Keywords: Simulation; Healthcare; Colonoscopy; Ergonomics; Training 


\section{Ergonomics International Journal}

\section{Introduction}

This research investigates the use of the Kagaku model, a newly developed 'still' model to acquire the early technical skills of colonoscopy in the training program. Virtual reality simulators have been researched in this capacity and found to be effective in enhancing learning of the technical skills of colonoscopy, transferring to increased patient performance [1,2]. However, the high cost and limited accessibility of these virtual reality simulators limits their use in training. The Kagaku model provides a viable alternative, however has not yet been validated in the literature. This study will thus act as the first step to determine whether the model is effective at improving colonoscopy performance. This study will additionally compare the learning curve of the two model conditions (the closed condition with no view of the abdomen, and an intermittently open condition with a view of the abdomen) to determine if one condition produces a higher performance than the other. With a validated model used at the beginning of the training program, trainees can acquire the technical skills of colonoscopy so they become automatic prior to entering the operating room, thus reducing the learning curve to achieve competence and reducing the associated training time, cost and resources required. Trainees can then perform colonoscopies on patients with a higher initial skill level resulting in better patient care. In addition, trainees will have free mental workload available to maximise the use of patient-based training and deal with complex cases under the supervision of an experienced colonoscopist [3].

Colonoscopies are usually performed in a hospital or day clinic under various levels of sedation. Patient discomfort reported from the procedure varies with the amount of sedation used, an individual's threshold for pain, colon characteristics and the colonoscopist's skill [4]. The risk associated with a colonoscopy is also associated with colon characteristics and the colonoscopist's skill, where thin colons with difficult shapes to manoeuvre are more likely to tear, and less experienced colonoscopists are more likely to push inward with the scope, applying increased force on the colon wall, increasing the risk of tearing [5]. Despite the risks involved in colonoscopy, there is a low complication rate of $0.14 \%$, comprising largely of bleeding and perforation complications [6].

A typical colonoscopy set-up is displayed in Figure 1. Most colonoscopists place the patient in the left lateral position for the procedure [7]. The equipment to perform the procedure involves a colonoscope (scope), light source, connections for suction and water, and a monitor, displaying the luminal view of the colon obtained from the scope tip. A control stand powers the scope functions, and projects the image collected from the scope onto the monitor.

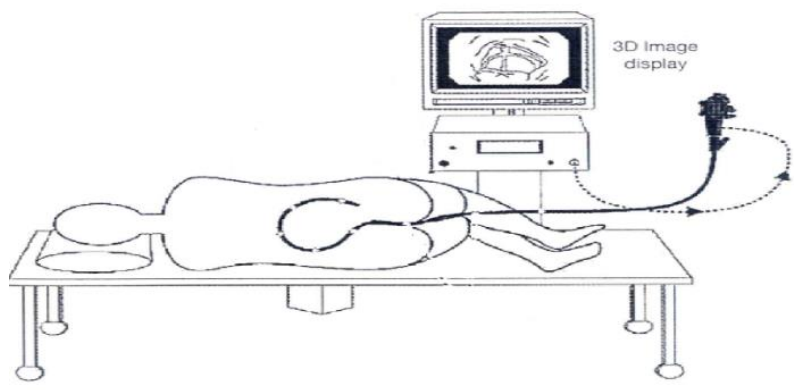

Figure 1: Colonoscopy set-up.

The scope has three main sections; a flexible shaft with a manoeuvrable tip, a control head, and an umbilicus which is connected to the colonoscope stand [5]. The tip of the flexible shaft, contains a wide lens (90-130 degree) camera and light source used to capture an image of the colon, (a few millimetres to around 15 centimetres, depending on the scope position), which is displayed on the monitor for the colonoscopist to use to guide navigation and diagnosis. The tip of the scope can then be manoeuvred in the lateral and horizontal position, using the two dials on the control head. The colonoscopist will typically hold the scope in their left hand, using their left hand figures to manipulate the controls in the desired direction, refer to Figure 2. The scope was originally designed as a two-handed operation, and thus requires an awkward single-single grip in operation to free the other hand to control the shaft [5] The colonoscopist holds the shaft of the scope with their right hand approximately $20 \mathrm{~cm}$ from the tip of the shaft with gauze used for grip, and inserts the tip of the scope into the anus. By gradually applying force to the scope, the scope will progress into the colon.

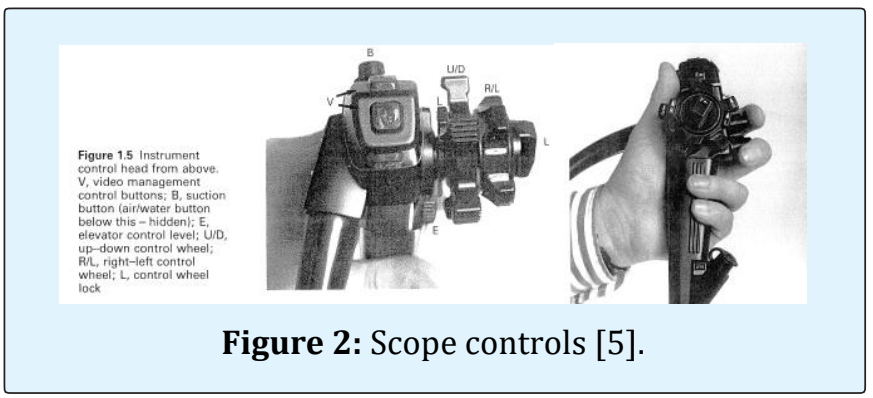




\section{Ergonomics International Journal}

Inserting the scope into the colon requires a high level of technical skill. The natural shape of the colon contains multiple turns, fixed ascending and descending colon sections, while the remaining sections are somewhat free moving, which makes it difficult to advance the scope by simply pushing inward Figure 3a [8].

In addition, there are vast individual differences in colon lengths and shapes. Older adults and other clinical positions have thinner colon walls, and are therefore at increased risk of perforations. Females have longer colons and are more difficult to navigate. Some individuals have congenital colon mal-rotations, such as Alpha loop and Gamma loop configurations, refer to Figure 3b / Figure 3c respectively [9]. These characteristics will be unknown to the colonoscopist, thus the colonoscopist must rely heavily on their mental models, experience, and perception of cues to determine the colon shape.

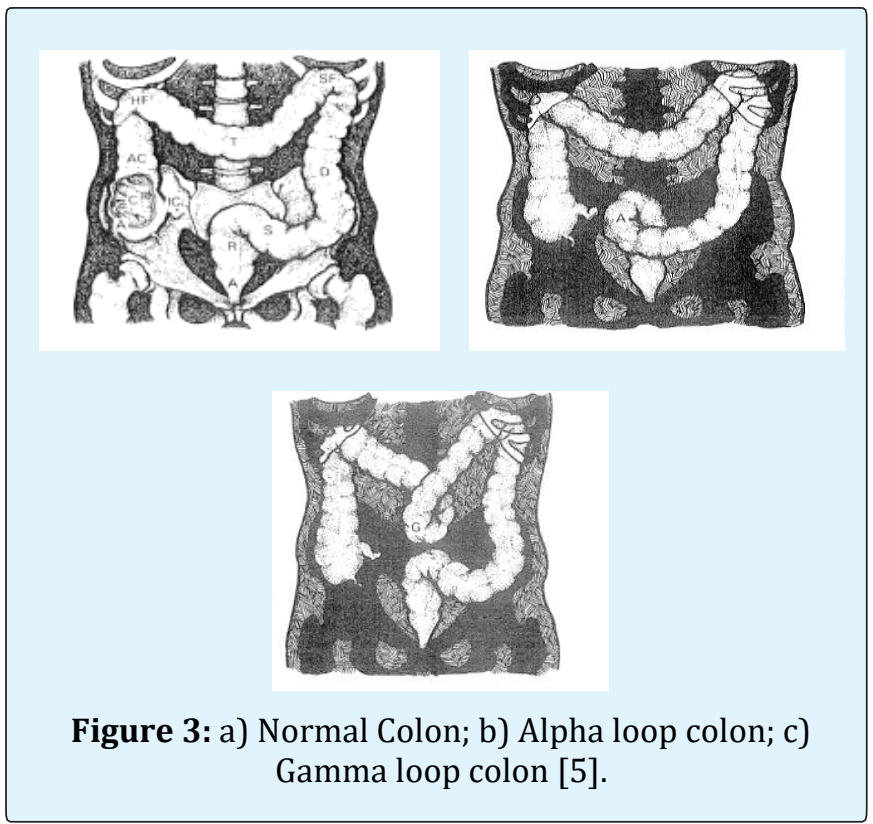

The major technical difficulty in colonoscopy is dealing with the flexible shaft 'loops', which spontaneously form when pushing the scope into the colon. 'Loops' involve the scope tip failing to advance further into the colon, instead resulting in stretching the bowel wall, causing patient discomfort and risk of perforation, refer to Figure 4 [5]. The formation of 'Loops' is caused by the combination of the curvy anatomical shape of the colon and the stiffness of the scope [10].

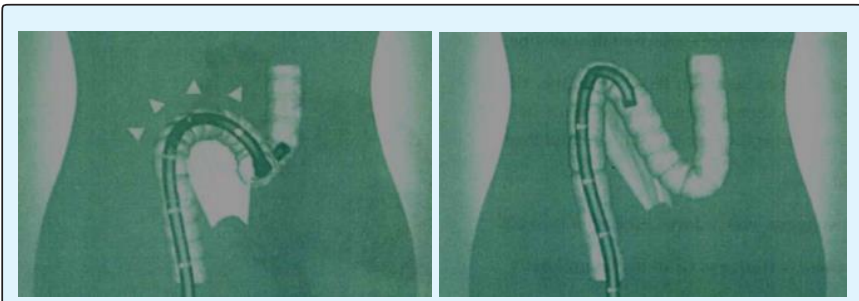

Figure 4: The formation of a loop.

\section{Performing a Colonoscopy}

A colonoscopy involves two major phases: the insertion phase, involving the technical skill of intubating the scope until the end point of the colon is reached (caecum), and the withdrawal phase, involving the careful diagnosis of pathology and treatment of any abnormalities [5]. By dividing the technical and cognitive components of colonoscopy into two phases each can be performed to a higher degree of accuracy. The technical phase of intubation will be the focus of this paper.

A hierarchical task analysis was conducted by a team of researchers that identified the operations expert colonoscopists performed to achieve the overall goal of intubation of safely reaching the caecum, Figure 5 [11]. Scope handling refers to steering using the shaft and control body dials, performing three main steering operations: manoeuvring the dials, the transmission of twist from the control head to the tip by wrist action and twisting the shaft with the right hand in the right direction and using the angulation controls. Situational awareness refers to the information processing required to attend, perceive and comprehend the stimuli from the environment, and comparing this with knowledge structures about colonoscopy, to maintain an accurate mental model of the scope tip position, relative to the colon [12].

Despite the intubation phase being largely technical, in practice colonoscopists will observe and perceive any abnormalities that they encounter using their clinical skills, and will examine thoroughly during the withdrawal phase. Due to the technical demands of colonoscopy, specific non-technical skills such as dealing with stress, also aid in successful intubation. These operations are performed interdependently: for example, a colonoscopist must have a high situational awareness to perform the correct scope handling techniques. 


\section{Ergonomics International Journal}

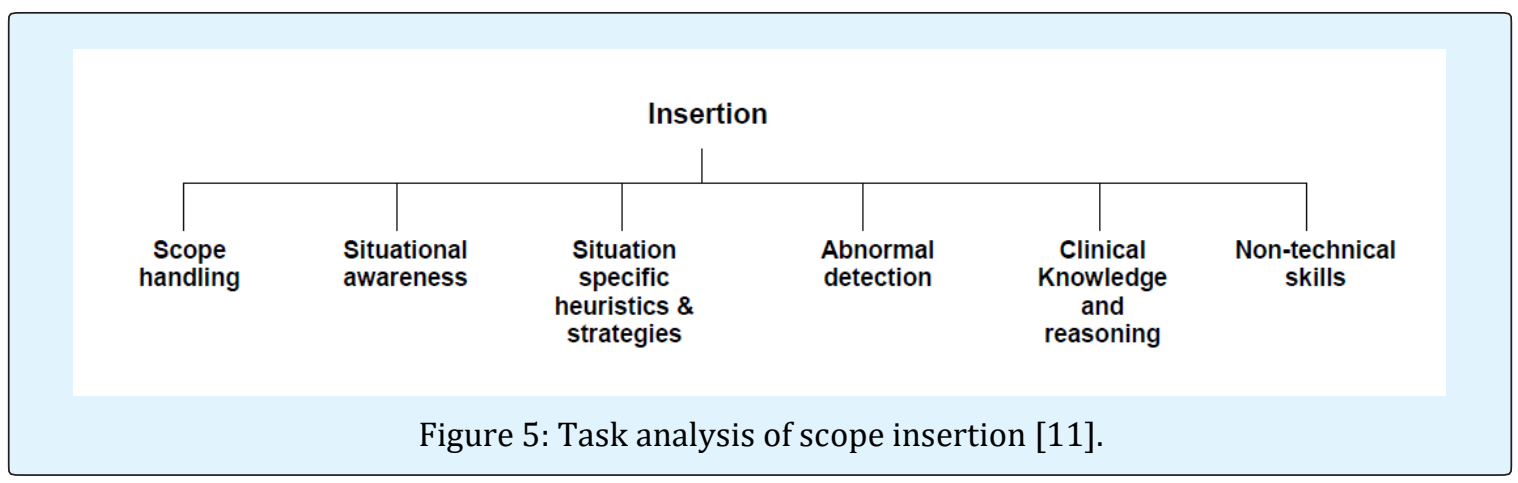

Gaining colonoscopy proficiency is difficult [5]. Novice colonoscopy trainees simply push inward, making the loop bigger, and the patient more uncomfortable. It takes a long time to develop scope handling, situational awareness and situational specific heuristics and strategies required to achieve quick, intubation with minimal patient discomfort. The current approach to training is based on the traditional Halstedian apprenticeship model of patient-based one-on-one instruction, lacking a formal educational context $[9,13]$. Training involves performing colonoscopies on patients with a colonoscopist providing instructions throughout the procedure. Usually training occurs once or twice a week, for a period of approximately two years. The American Society of Gastrointestinal Endoscopy (ASGA) recommends trainees must perform a minimum of 100 unassisted, supervised, completed, colonoscopies to achieve 'competency', where the caecum is reached in $90 \%$ of cases, before colonoscopists can practice independently [14]. However, literature revealed that colonoscopists may be practicing independently following this criteria with substandard competence as it was found that approximately 200-300 procedures were required to reach the caecum in $90 \%$ of cases [14]. This finding reveals that the Halstedian method of training results in an extremely long learning curve, which may not be efficient at training trainees to the required level of competence. It has also been anecdotally reported in the literature that this method of training is frustrating for both the trainee and supervisor [5]. Training on patients also may not be optimal in terms of patient care. Trainees, particularly in early learning are likely to use cruder motor movements and greater force to advance the scope through difficult anatomy causing patient discomfort and risk of perforating the bowel wall, which can result in patient death [15].

The Halstedian method of colonoscopy training is based on the premise that whole-task practice improves trainee performance, a theory largely supported by traditional motor learning literature. However, most of these early studies involve simple motor tasks. Colonoscopy, moreover, involves a complex task as it involves high movement complexity, has a large number of possible response errors and variability in movements [16]. More recent studies involving complex motor tasks, have found that the whole-part methods of training can lead to lower performances and a resulting longer learning curve to achieve expertise $[13,17]$. This finding can be explained by workload theory, which stipulates that humans have a finite capacity to process information [18]. The whole-part method of practice involved in the Halstedian method of training colonoscopy thus places large demand on the processing capacity of the trainee. Trainees have to attend to multiple stimuli such as the visual information on the monitor and haptic information from the scope and the controls. These demands on attention can exceed the capacity of the novice and lead to mental overload [19]. Mental overload can lead to resulting performance degradations following the YerkesDoson law [20]. Performance decrements can also result in an increase in errors and perceptual/attention narrowing where the range and breadth or attention to concentrate on one thing is diminished [21].

The Halstedian method of clinical training is also based on the premise that instruction improves performance. This notion is the foundation of the coaching concept prevalent in the motor skill acquisition literature surrounding sports. However, this has been found in more recent literature not to be beneficial is all cases [17]. It is found that instructions may disrupt rather than facilitate learning. With the high workload of performing colonoscopies, it is likely that trainees do not have any capacity to attend to the instruction of the supervising colonoscopist. In addition, the presence of the colonoscopist may also increase the stress of the trainee, which contributes to the high mental workload and performance detriments. Instructional messages provided may be too complex and presented in language which is 


\section{Ergonomics International Journal}

difficult for the trainee to perceive and comprehend. This is likely for colonoscopy, where many colonoscopists have difficulty verbalising the skills they use [5]. In addition, one coaching review found that instructions that specify the 'best' method for performing a skill have little benefit [22]. This is particularly true for colonoscopy where the optimal method for performance varies and cannot always be generalised across individuals. In the Halstedian method of training, there is also less scope for trainees to learn via the problem-solving process which has been found to be an essential component of motor learning [17]. This is not possible, as the trial and error methods involved in training on patients can result in patient discomfort and bowel wall perforation, which can lead to death.

\section{Simulators}

In an effort to standardise and optimise learning, researchers have shown an increased interest in the roles of simulators in the teaching of at least some of the most basic surgical skills across various medical disciplines $[23,24]$. Simulators provide an alternative training method to practice the technical tasks and skills repeatedly until proficiency is achieved [5]. Simulators take a streamlined approach to skill acquisition, which refers to performing a simplified version of the whole skills of colonoscopy. This method of learning has been found to be effective for learning complex skills, such as colonoscopy [19]. Simulators can be used independently of a trainer and allow for trial and error learning, which has been found to be effective in acquiring complex and novel skills such colonoscopy [25]. Simulators segment the skills of colonoscopy to train the technical skills alone. With practice, the functional complexity of the technical skill will reduce, as the processing demands decrease and the process becomes more automated. This automation can be observed by smaller response errors, reduced attentive demands and increased movement efficiency [19]. When the technical skills of colonoscopy become automated, the tasks can be performed with little conscious attention, allowing the trainee to allocate attention to tasks that require more cognitive resources, such as acquiring the clinical skills in patients in subsequent training [18]. The use of simulators in a training regime allows for individual differences in automation times and for individuals to reduce the times between training sessions, thus reducing the time to achieve competence. In addition to the learning benefits of simulators of trainees gaining technical skill proficiency, simulators will potentially reduce the role of the colonoscopy supervisor up to $10-37 \%$ [1]. This results in a predicted saving of $\$ 500,000$ to $\$ 1,000,000$ per training year.

Various simulators have been developed over the past twenty-five years in an attempt to produce a realistic representation of colonoscopy. The majority of research has been dedicated to virtual reality (VR) models such as the GI Mentor 2 and Accu Touch, refer to Figure 6. These simulators are equipped with an authentic endoscope, tactile force feedback system, a library of clinical cases for each procedure, varying difficulty levels, feedback from the patient (about levels of pain experienced), the ability to incur complications and a monitor of vital signs [1]. VR Simulators have been found to distinguish between experts and novices, reduce total examination times, allow recognition of pathology, recognise patient discomfort, and reduce time spent colliding with the bowel wall [2]. In addition, they can transfer skills acquired to performance on patients [1]. The major limitation of virtual reality simulators however, is their high cost and limited availability, which reduces their training utility $[2,26]$.

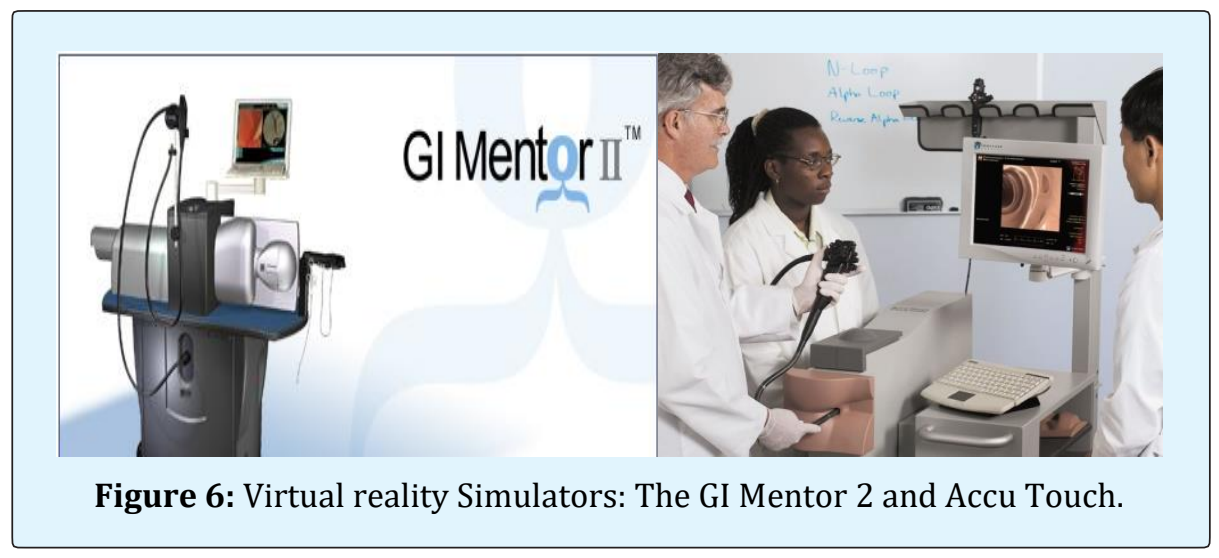

Hannah M. The Use of Simulation in Training to Improve Motor Learning Skill Acquisition for Complex Tasks in the Medical Industry. Ergonomics Int J 2019, 3(3): 000205. 


\section{Ergonomics International Journal}

\section{Use of Simulation}

A newly developed simulator is the Kyotokagaku-M40 (Kagaku) model (refer to Figure 6) which holds promise as a low cost alternative simulator. The soft, flexible and airtight material it is manufactured from allows realistic colonoscope insertion and withdrawal, as well as difficult manoeuvre techniques. It allows multiple colon variations and is potentially high for realism and moderate for difficulty. There is evidence that low fidelity bench top models are as effective as high-fidelity models [27]. These models improve cost effectiveness and maintain the learning objectives.

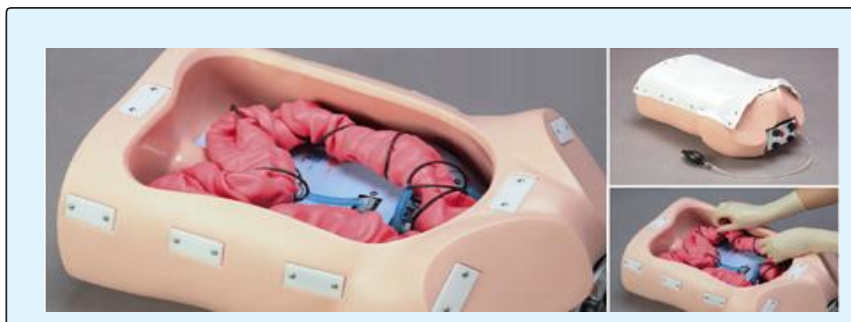

Figure 6: Kyotokagaku-M40 Colonoscopy Training Model.

An additional benefit of the Kagaku model is that it can be used in two conditions; an open (lid off) and closed (lid on) condition. The open condition allows additional visual feedback of the scope position inside the colon, as the scope in the light tip illuminates the corresponding section of the colon. This allows for trainees to maintain a mental model of the task. In addition, this form of visual feedback has been found to improve the learning in motor skill acquisition across motor learning literature in sports tasks [28]. The open condition allows for situational awareness to be maintained when a difficult loop is faced. The closed condition on the other hand, has the added realism of a colonoscopy procedure, where no visual feedback is present. With the early technical skills of colonoscopy acquired on the Kagaku model, trainees can potentially enter the operating room to perform a higher quality of colonoscopy on patients with subsequent training and reduce the time to reach colonoscopy competency.

\section{Relevance of the Project}

This study will pave the way in the development of an alternative training regime to acquire the technical skills of colonoscopy. Studies investigating colonoscopy simulators adopt small sample sizes therefore this study will further support the use of simulators as a training tool with improved statistical validity. This study will be the first to examine the utility of the Kagaku model as a training tool to acquire initial technical proficiency in colonoscopy. Although virtual reality simulators have been studied in depth in the literature, they are of high cost and have limited accessibility, therefore could not be implemented in a training regime standardized across medical institutions [1]. This research seeks to validate the Kagaku model as a training tool to enhance performance over time. Performance will be measured by the time taken to reach various anatomical positions in the colon. In addition, this study will investigate whether the Kagaku model can be better utilized in its open condition compared with its closed condition. The open condition allows the trainee to view the dynamic colon, and the light from the scope tip illuminating the approaching section of colon. The concept of enhancing learning through the additional feedback mechanism of visual feedback on the colonoscopy tip relative to the colon configuration, to enhance colonoscopy training has not been researched. By investigating alternative training mechanisms for learning, this can provide insight into the skill acquisition process in colonoscopy. With the Kagaku model used to acquire the technical skills of colonoscopy, patient care will increase and the time for trainees to achieve competency will also be reduced. This will potentially increase the colonoscopist resources. With resources closer to meeting demands of the follow-up screening initiative of the Australian Government, the detection and survival rate of colorectal cancer can be improved.

The main aim of this study is to investigate whether training on the Kagaku model improves performance over time. An additional aim of the study is to investigate the performance differences between trainees who receive training in the Kagaku intermittent condition, where participants can view the colon configuration after five minutes of it closed compared with the Kagaku closed condition where the colon configuration remains unknown after training. Participants were randomly assigned to either the Kagaku 'open' or 'intermittent' condition. The closed group must never see into the model. The research assistant (RA) controls when the intermittent group see into the model via removing the occluder five minutes into each training session. Both groups received the same training program involving two, one-hour sessions a week, over nine-weeks, practicing the skills of colonoscopy, with increasingly difficult colon configurations. Novices' performance on the simulator was tested before and after the training sessions in the Kagaku model closed position with various colon cases (configurations). The performance measures will include the time taken to reach various colon anatomical locations. 


\section{Ergonomics International Journal}

It was predicted that the time taken to reach the various anatomical locations would decrease across trials, in both training conditions, in both the easy and difficult cases. It was predicted that the performance on the difficult cases requiring loop reduction techniques would be greater for individuals who received training in the intermittent condition after training. It was predicted that there would be no difference in performance across intermittent and closed groups before training. It was also predicted that there would be no differences between intermittent and closed groups for the easy case with no loop reduction required.

\section{Methodology}

\section{Participants}

Sixteen first year medical students from the University of Queensland (14 male) participated in the experiment. Participants ranged from 19 to 34 years old $(M=24.88$, $S D=4.07)$. Participants were sourced through the University of Queensland recruitment through an expression of interest. Participants were offered $\$ 220$ dollars to be paid upon completion of the total sessions in the 9-week training program. Participants with prior colonoscopy knowledge were excluded from expressing interest, to ensure that no performance confounds were present. Participants were randomly assigned to Kagaku model groups (open and intermittent). Demographic information of handedness, height and sex were collected prior to testing to ensure no initial confounding factors existed. The age of participants between groups was not statistically significant $(t=.25, p=.80)$ and handedness and gender was balanced across groups.

\section{Design}

The study adopted a mixed type design. The between group variables were the Kagaku model group, operational across two levels: closed (no direct view of the abdomen) and intermittent (occasional views into the abdomen). The first within group variable was the Kagaku colon configuration, specified by the 'Case number', operationalised across four variables: Case 2, Case 3, Case 4 and Case 6. The second within group variable was training, operational across two levels: before and after training. It is noted however, that Case 4 and Case 6 were only performed in the after training level, as it was predicted that participants would not be able to progress very far into the colon before training in these difficult cases, thus would not be a useful outcome measure. Refer to Table 1 for the testing and training schedule. The dependent variable was the time taken to reach five anatomical landmarks in the closed condition (Recto sigmoid, sigmoid-descending, splenic flexure, hepatic flexure, caecum).

\begin{tabular}{|c|c|c|c|}
\hline Day & Session Type & Case & Instructional Video \\
\hline 1 & Training & Case 0 + one training session & Video 1 \\
\hline 2 & Testing & Case 2 & Video 2 \\
\hline 3 & Testing & Case 3 & Video 3 \\
\hline 4 & Training & Case 3 & Video 4 \\
\hline 5 & Training & Case 3 & \\
\hline 6 & Training & Case 3 4 - Case 4 and clip & Video 4 \\
\hline 7 & Training & Case 4a - Case 4 and clip & Video 4 \\
\hline 8 & Training & Case 5a - Case 5 & \\
\hline 9 & Training & Case 5a - Case 5 & \\
\hline 10 & Training & Case 6 & \\
\hline 11 & Training & Case 6 & \\
\hline 12 & Training & Case 4 & \\
\hline 13 & Training & Case 4 & \\
\hline 14 & Training & Case 6 & \\
\hline 15 & Testing & Case 4 & \\
\hline 16 & Testing & TEST Case 3 & \\
\hline 17 & Testing & TEST Case 2 & \\
\hline
\end{tabular}

Table 1: Schedule of Experiment. 


\section{Ergonomics International Journal}

\section{Results}

Participant data was compiled and analysed to see if there was a difference between the before and after training, and closed and intermittent groups, on the average time it took to reach the five colon anatomical landmarks (recto sigmoid, sigmoid-descending, splenic flexure, hepatic flexure, caecum) across colon cases (Case 2, Case 3, Case 4, Case 6). A series of AVOVA's were conducted by way of SPSS' General Linear Model Profile Analysis, due to the nature of the mixed design (a 2 level grouping factor: closed and intermittent colon groups, a four level. Note Cases 4 and 6 were only performed in the post training condition. Some qualitative analysis of the findings will also be considered for the development of future research directions.

As there were large numbers of missing data points on the trainee's first testing case (Case 2, Trial 1), and first trial at of a more difficult case (Case 3, Trial 1), due to persons not being able to reach anatomical positions within the 20 minute time constraint, data from this trial was deleted. For trainee's second testing case for these cases (Case 2, Trial 2 and Case 3, Trial 1), there were also missing data points thus their scores were replaced with a dummy value, determined by the gradient of the learning curve of the mean of the group, for that location.

To test whether there were any initial skill difference in participants in the open and intermittent groups on Case 2 (requiring no loop reduction) and Case 3 (requiring loop reduction), across the dependent measures of reaching the five anatomical locations (recto sigmoid, sigmoid-descending, splenic flexure, hepatic flexure, caecum), ANOVA's were performed by way of SPSS' General Linear Model Profile Analysis, due to the nature of the mixed design (a 2 level grouping factors, intermittent and closed, and the time it took to reach the various anatomical location, and a five level withinsubjects factors, time across trials). It was revealed that there was a non-significant main effect between the open and intermittent groups across locations and cases, $F(1,14)$ $0.003, p=0.96$. There was non-significant interaction between group and location $F(1,14)=.03, p=.10$ and a non-significant interaction between case and group and location, $F(4,12)=.09, p=.10$. This indicates that there were no initial differences between groups in both technical and loop reducing abilities refer to Figure 7 and Figure 8.

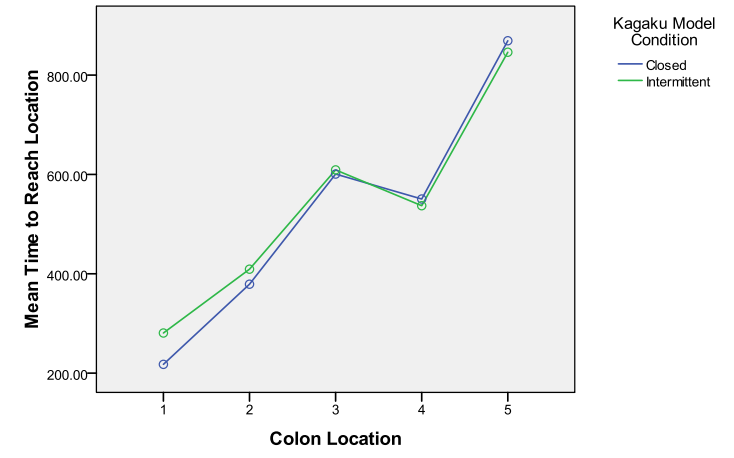

Figure 7: Mean Group Differences on Case 2 Posttraining.

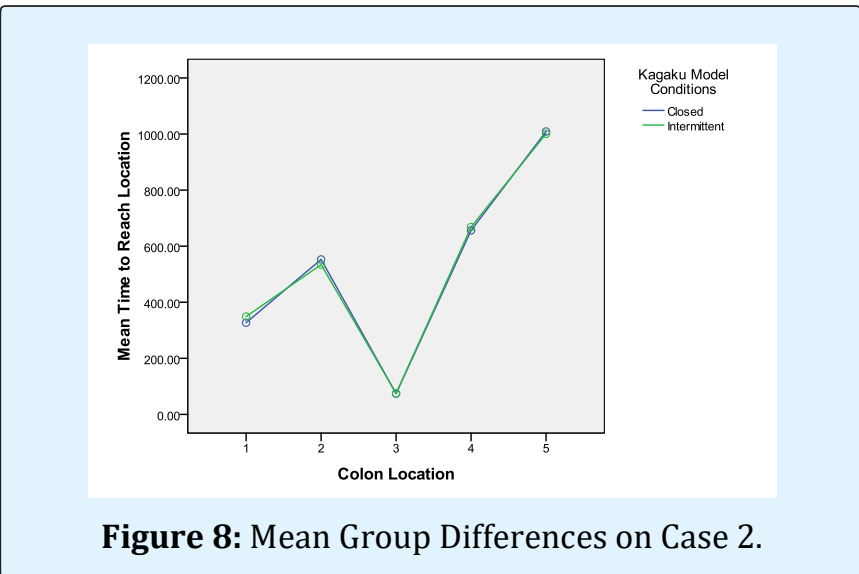

Training was the second independent variable to be assessed by profile analysis. Training was firstly examined for Case 2, the case requiring no loop reduction component. To test whether there were any performance differences before and after training across the dependent measures of reaching the five anatomical locations (recto sigmoid, sigmoid-descending, splenic flexure, hepatic flexure, caecum), ANOVA's were performed by way of SPSS' General Linear Model Profile Analysis, due to the nature of the mixed design (2 level grouping factors, before and after training, and the time it took to reach the various anatomical location, and five level within-subjects factors, time across trials). It was revealed that there was a significant main effect for training across groups and locations, $F(1,14)=13.07, p=.00$, where participants performed significantly better across all locations after training than before training. There was also a significant Interaction between training and location, $F(4,12)=29.20$, $P=.00$, refer to Figure 9 . 


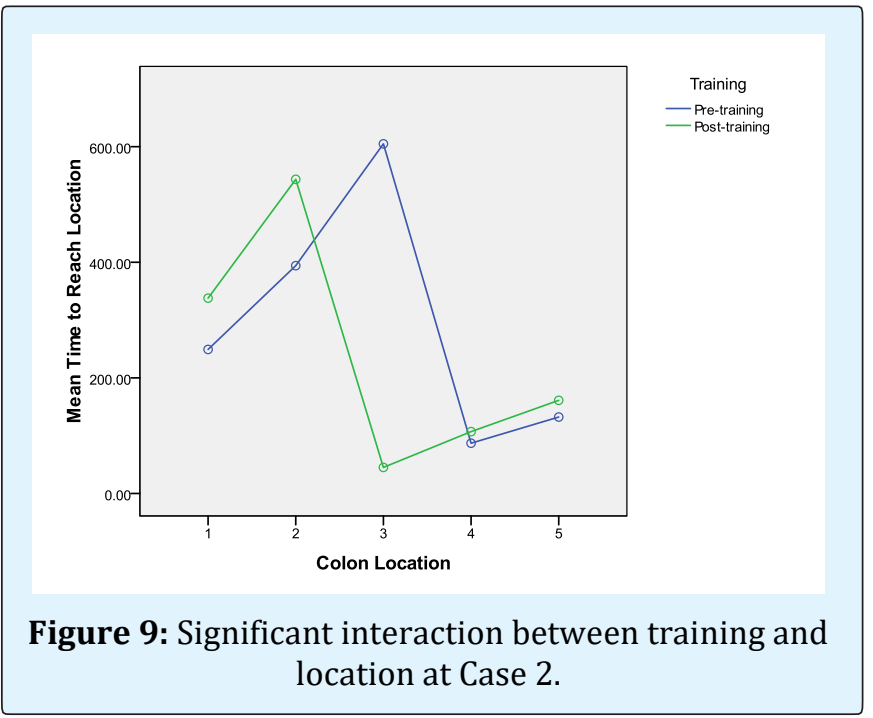

A series of follow-up repeated measures t-tests were conducted to determine differences between pre-training and post-training conditions for each of the five colon locations, refer to Table 3. It was revealed that there was a significant difference for all of the colon locations, where times taken to reach the locations after training was less than before training.

\begin{tabular}{|c|c|c|c|}
\hline & $\boldsymbol{t}$ & $\mathbf{d f}$ & $\boldsymbol{p}$ \\
\hline Location 1 & 2.956 & 15 & 0.01 \\
\hline Location 2 & 3.016 & 15 & 0.01 \\
\hline Location 3 & 3.337 & 15 & 0.01 \\
\hline Location 4 & 4.451 & 15 & 0 \\
\hline Location 5 & 4.65 & 15 & 0 \\
\hline
\end{tabular}

Table 3: T-tests of Case 2 Locations Before and After Training.

Training was the second independent variable to be assessed by profile analysis. Training was firstly examined for Case 3, the Case with an alpha loop. To test whether there were any performance difference before and after training across the dependent measures of reaching the five anatomical locations (recto sigmoid, sigmoid-descending, splenic flexure, hepatic flexure, caecum), ANOVA's were performed by way of SPSS' General Linear Model Profile Analysis, due to the nature of the mixed design (2 level grouping factors, before and after training, and the time it took to reach the various anatomical location, and five level within-subjects factors, time across trials). It was revealed that there was a significant main effect for training across groups and locations, $F(1,14)=6.24, p=.03$, where participants performed significantly better across all locations after training than before training. There was also a significant Interaction between training and location, $F(4,12)=42.88$, $P=.00$, refer to Figure 10 .

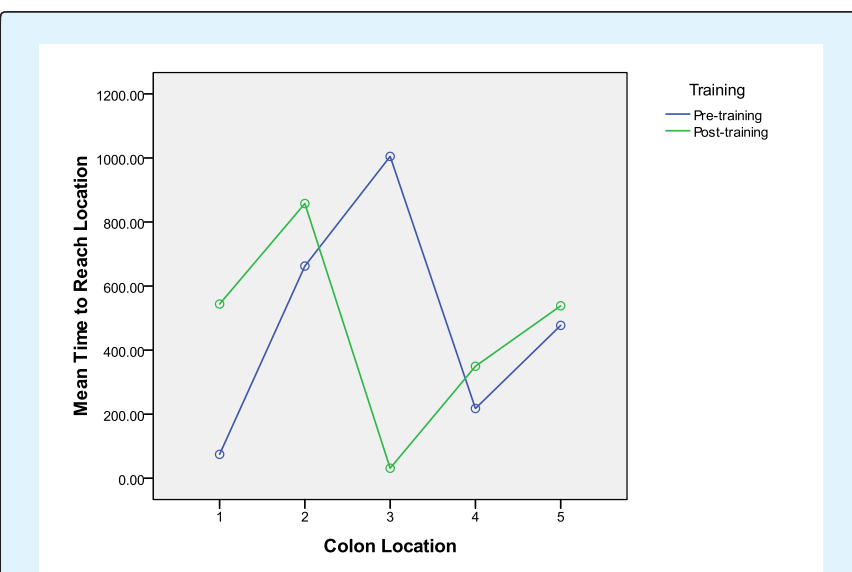

Figure 10: Significant interaction between training and location at Case 3.

An ANOVA was performed to compare the open and closed groups on the time it took to reach the various anatomical locations in the colon across cases (2 level grouping factors, intermitted and closed, and the time it took to reach various anatomical location $s$ across the four cases which involved looping 3, 6, and 4). It was revealed that, there was a significant two-way interaction between location and training $F=16.37, \mathrm{P}=0.00$. There was a significant three-way interaction between training, location and case, $\mathrm{F}=35.084, \mathrm{p}=.00$. There was a significant three-way interaction between location, training and model, $F=3.910, p=.007$. There was a nonsignificant interaction between location, training, case and model, $\mathrm{F}=0.804, \mathrm{p}=5.28$.

A series of follow-up repeated measures t-tests was conducted to determine differences between pre-training and post-training conditions for each of the five colon locations, refer to Table 4. It was revealed that there was a significant difference between training across all locations, where times taken to reach the locations were significantly less after training than before training

\begin{tabular}{|c|c|c|c|}
\hline & $\boldsymbol{t}$ & df & $\boldsymbol{p}$ \\
\hline Location 1 & 2.956 & 15 & 0 \\
\hline Location 2 & 3.016 & 15 & 0.01 \\
\hline Location 3 & 3.337 & 15 & 0.02 \\
\hline Location 4 & 4.451 & 15 & 0.01 \\
\hline Location 5 & 4.65 & 15 & 0 \\
\hline
\end{tabular}

Table 4: T-tests of Case 3 Locations Before and After Training. 
An ANOVA was performed to compare the open and intermittent groups on the time it took to reach the various anatomical locations in the colon across cases (a 2 level grouping factors, intermitted and closed, and the time it took to reach various anatomical location $\mathrm{s}$ across the four cases 2, 3, 6, and 4). It was revealed that, there was a non-significant main effect for group condition $F(1,14)=.00, p=.78$. There was also a non-significant three-way interaction between location, case and model $F(12)=.67,, p=.78$.

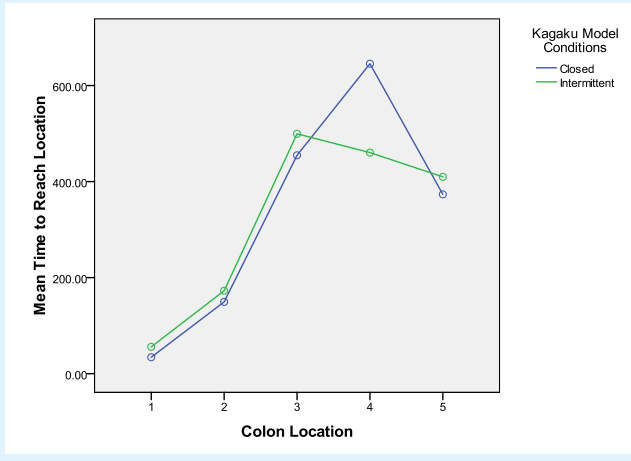

Figure 11: Case 2 Model Groups After Training.

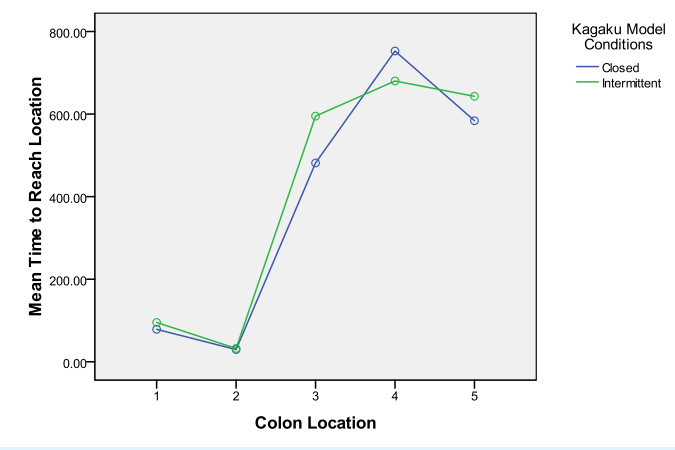

Figure 12: Case 3 Model Groups After Training.

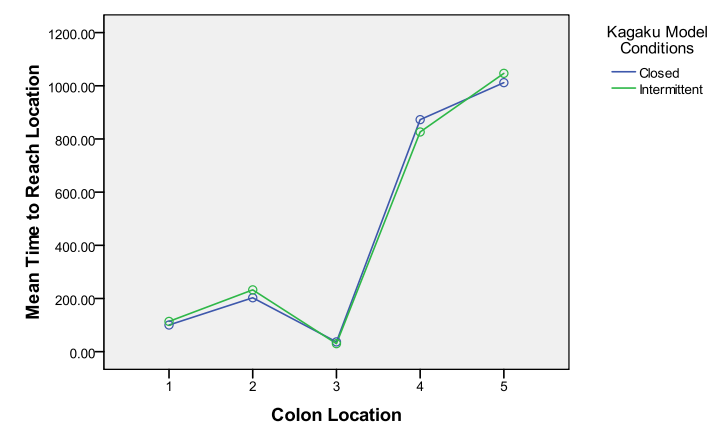

Figure 13: Case 6 Model Groups After Training.

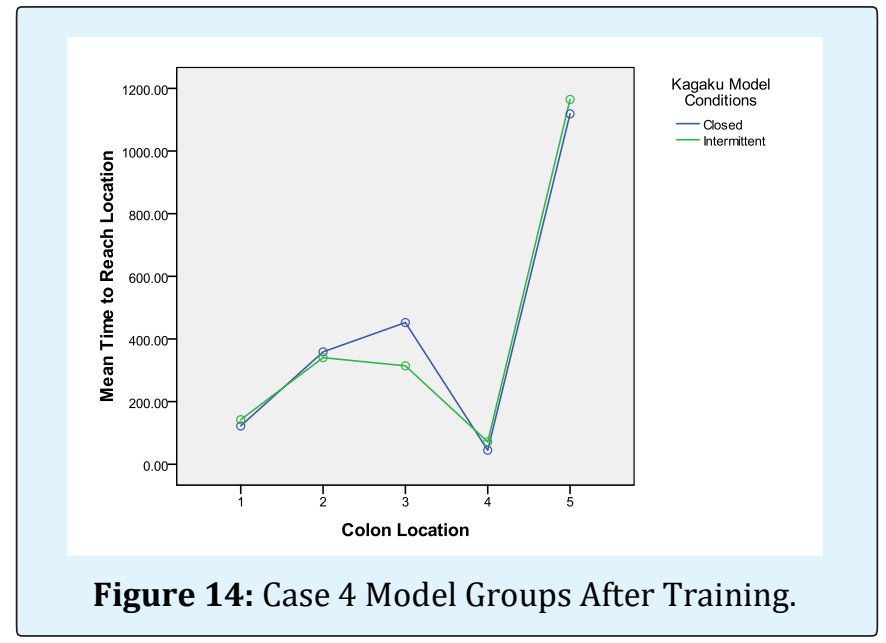

\section{Discussion}

This study investigated the utility of the Kagaku model as a training tool to acquire the technical skills of colonoscopy. This study also investigated whether performance and learning would be enhanced in the intermittent Kagaku model condition with additional visual feedback compared with the closed condition. Performance was measured during four testing sessions using both a difficult and easy case in the closed model condition time to reach various anatomical landmarks. It was hypothesized that performance in both groups would improve across training sessions. A series of ANOVA's were conducted on the data where it was found that the Kagaku model can be used to improve performance across trials, which supported the hypothesis. However, there were no differences between model conditions after training on any of the cases.

\section{Training Effects}

This study validated the Kagaku model as an alternative method to acquiring the initial technical skill acquisition of colonoscopy to the Helstedian traditional approach to training. It provided an initial step into understanding the rate of learning of trainees on the model. It was qualitatively demonstrated that similar to motor learning studies in sports, the learning curve was initially steep, however became shallower with training. This change in learning curve gradient occurred quickly, after a few weeks of training. This provides some insight into the potential training regime of colonoscopy. The high frequency of training using a simplification model of the whole-part skill of intubation, with a gradual increase in the difficulty of cases, may accelerate the acquisition of colonoscopy skill. This follows practice theory, where 


\section{Ergonomics International Journal}

with increased practice, increased performance results [22]. In addition, it follows the recent findings that simplification can enhance learning of complex skills [19]. It was qualitatively noted that the small errors and increased movement efficiency achieved at the later stages of learning, indicated that the technical skill of scope handling became automated, and the specific skills and heuristics of the trainee had developed to allow for loop reduction techniques to be applied when various loop situations were encountered [29-34].

\section{Conclusion}

Despite the limitations of this study, it is apparent that the results further support the literature findings of the benefits of simulator training on early motor skill acquisition. This study set out to validate use of the Kagaku model as a training tool, and the results demonstrated that with practice on the simulator, performance improvements were made. In addition, this study found that there were no differences in performance after training in the Kagaku model open and intermittent conditions. While the practical utility of the Kagaku model is still lacking due to the limitations of the study, student sample used and the potential confounding variables in the training regime such as training schedule, this study has provided a starting point for further investigation into colonoscopy training. With this study, future studies can refine the colonoscopy training regime to optimise technical skill learning using simulators. Future studies can then investigate its utility to improve performance in a clinical setting. This study has provided the first step into the development of an improved training regime to acquire colonoscopy expertise. This method of analysis using simulators can be adopted to investigate the benefits of the use of simulators in training for a wide range of complex tasks.

\section{References}

1. Gerson L (2006) Can colonoscopy simulators enhance the learning curve for trainees? Gastrointestinal Endoscopy 64(3): 369-374.

2. Sedlack R, Kolars J (2003) Computer simulator training enhances the competency of gastroenterology fellows at colonoscopy: results of a pilot study. American Journal of Gastroenterology 99(1): 33-37.

3. (2001) Basic Skills of Colonoscopy. Raven Department, Royal College of Nursing.
4. Lee SH, Chung IK, Kim SJ, Kim JO, Ko BM, et al. (2008) An adequate level of training for technical competence in screening and diagnostic colonoscopy: a prospective multicentre evaluation of the learning curve. Gastrointestinal Endoscopy 67(4): 683-689.

5. Baillie J (1997) Gastrointestinal Endoscopy: Beyond the Basics. Butterworth Heineman, Oxford.

6. Austoker J, Hewinston P (2008) Screening for Colorectal Cancer. BMJ.

7. Waye J, Rex D, Williams C (2003) Colonoscopy principles and practice. Blackwell Publishing, Massachusetts.

8. Beart R, Fleshman J, Jacobson K, Levy J, Pemberton J (2005) Anatomic problems of the colon. National Digestive Diseases Information Clearinghouse.

9. Thomas-Gibson S, Williams C (2005) Colonoscopy training-New approaches, old problems. Gastrointestinal Endoscopy Clinics of North America 15(4): 813-827.

10. Buzink SN, Koch AD, Heemskerk J, Botden SM, Goossens RH (2007) Acquiring basic endoscopy skills by training on the Gi Mentor 2. Surgical Endoscopy 21(11): 1996-2003.

11. Zupanc C, Burgess-Limerick R, Watson M, Riek S, Wallis G, et al. (2009, October). A colonoscopy Competency Framework Derived from Task Analysis. Presented at Gastrointestinal Week, Sydney, Australia.

12. Endsley M (1995) Towards a theory of situation awareness in dynamic systems. Human Factors 37(1): 32-64.

13. Brydges R, Carnahan H, Backstein D, Dubrowski A (2007) Application of motor learning principles to complex surgical tasks: searching for ht optimal practice schedule. Journal of Motor Behaviour 39(1): $40-48$.

14. Marshall J (1995) Technical proficiency of trainees performing colonoscopy: a learning curve. Gastrointestinal Endoscopy 42(4): 287-291.

15. Shah G, Thomas-Gibson S, Lockett M, Brooker J, Thapas C, et al. (2003) Effect of real-time magnetic endoscope imagine of the teaching and acquisition of 


\section{Ergonomics International Journal}

colonoscope skills: Results from a single trainee. Endoscopy 35(5): 421-425.

16. Shah SG, Brooker JC, Williams CB, Thapar C, Saunders BP (2000) Effect of magnetic endoscope imagine on colonoscopy performance: a randomised controlled trail. The Lancet 356(9243): 1718-12722.

17. Thuraisingam AI, Levine DF, Anderson JT (2006) Can research in sports and other motor skills help improve endoscopy training. Gastrointestinal Endoscopy 63(2): 276-279.

18. Wickens C, Lee J, Liu Y, Becker S (2004) An Introduction to Human Factors Engineering. Pearson Education, New Jersey.

19. Wulf G, Shea C (2002) Principles derived from the study of simple skills do not generalize to complex skill learning. Psychonomic Bulletin \& Review 9(2): 185-211.

20. Yerkes R, Dodson J (1908) The relation of strength of stimulus to rapidity of habit formation. Journal of comparative Neurological Psychology 18: 459-482.

21. Driskell J, Salas E (1996) Stress and human performance. Lawrence Erlbaum Associate, Publishers Mahwah, New Jersey.

22. Hodges NJ, Franks IM (2002) Modelling and coaching practice: the role of instruction and demonstration. Journal of Sports Sciences 20(10): 793-811.

23. Aggarwal R, Hance J, Darzi A (2004) Surgical education and training in the new millennium. Surgical Endoscopy 18(10): 1409-1410.

24. Aggarwal R, Moorthy K, Darzi A (2004) Laparoscopic skills training and assessment. British Journal of Surgery 91(12): 1549-1558.

25. Wulf G, Weight C (1997) Instructions about physical principles in learning a complex motor skill: To tell or not to tell. Research Quarterly for Exercise and Sport 68(4): 362-367.
26. Cohen J, Cohen SA, Vora KC, Xue X, Burdick JS, et al. (2006) Multicentre randomised, controlled trail of virtual-reality simulator training in acquisition of competency in colonoscopy. Gastrointestinal Endoscopy 64(3): 361-368.

27. Grober ED, Hamstra SJ, Wanzel KR, Reznick RK, Matsumoto ED, et al. (2004) The educational impact of bench model fidelity on the acquisition of technical skill: the use of clinically relevant outcome measures. Annals of Surgery 240(2): 374-381.

28. Syed G Shah, Jim C Broker, Catherine Noriko S, Wukkuanbsm C, Sauders B (2002) Effect of magnetic endoscope imagine on patient tolerance and sedation requirements during colonoscopy: a randomised controlled trail. Gastrointestinal Endoscopy 55(7): 832-837.

29. Australian Government (2008) National Bowel Cancer Screening Program Information Booklet. Commonwealth of Australia.

30. Australian Bureau of Statistics (2005) 1303.0-Year Book Australia 2005 Cancer trends. ABS.

31. Australian Bureau of Statistics (2005) 4102.0 Australian Social Trends, 2005. Morbidity and Mortality: Colorectal Cancer. ABS.

32. Classen M, Tytgat G, Lightdale C (2002) Gastroenterological Endoscopy. Thieme Medical Publishers, New York.

33. Guadagnoli M (2004) Challenge point: A framework for conceptualising the effects of various practice conditions in the motor learning. Journal of Motor Behaviour 36(2): 212-224.

34. Koch AD, Buzink SN, Heemskerk J, Botden SM, Veenendaal R, et al. (2008) Expert and construct validity of the Simbionix GI Mentor 2 endoscopy simulator for colonoscopy. Surgical Endoscopy 22(1): 158-162. 